\title{
Basal Metabolism of Obese Adolescents: Evidence for Energy Conservation Compared to Normal and Lean Adolescents
}

\author{
VICTOR L. KATCH ${ }^{1}$, CHARLES C. MARKS ${ }^{2}$, M. DANIEL BECQUE ${ }^{3}$, \\ CATHERINE MOOREHEAD ${ }^{1}$, AND ALBERT ROCCHINI ${ }^{1}$ \\ ${ }^{\prime B}$ Behnke Laboratory for Body Composition Research, Department of Move- \\ ment Science, Division of Kinesiology, and Section of Pediatric Cardiol- \\ ogy, Department of Pediatrics-School of Medicine, University of Michi- \\ gan, Ann Arbor, Michigan 48109-2240; '2 Exercise Science Program, \\ School of Health Sciences, Oakland University, Rochester, Michigan \\ 48309; ${ }^{3}$ Department of Physical Education, Southern Illinois \\ University, Carbondale, Illinois 62901
}

\begin{abstract}
To test if obese adolescents systematically conserve energy, comparisons of basal metabolic rate (BMR) of obese, normal, and lean male and female adolescents were made. Obese had elevated values by as much as $23 \%(P \leqslant 0.05)$ expressed as $\mathrm{kJ} \cdot 24 \mathrm{hr}^{-1}$ compared to the normal and lean. When indexed to body mass $\left(\mathrm{kJ} \cdot \mathrm{kg}-\mathrm{BM}^{-1} \cdot \mathrm{hr}^{-1}\right)$, the BMR for the obese was depressed by as much as $-53 \%(P \leqslant 0.01)$, and when indexed to fat free mass $\left(\mathrm{kJ} \cdot \mathrm{kg}-\mathrm{FFM}^{-1} \cdot \mathrm{hr}^{-1}\right)$ it was depressed by $-33 \%$ compared to normal and lean adolescents. A "theoretical metabolic rate" (TMR), based on the observed fat free mass, fat mass, and their thermal equivalents, was proposed as a theoretical way to properly index basal metabolism, referenced to body composition. Comparisons of the TMR between the obese, normal, and lean revealed that the obese values were depressed by an average $-22 \%(P \leqslant 0.05)$. In comparison, differences in TMR between the normal and lean males and females were no larger than $8 \%$ (ns). It was concluded that since both the observed BMR (expressed relative to body composition), and the derived TMR values were depressed for the obese compared to the normal and lean adolescent, the data suggest an energy saving hypothesis for obese adolescents.
\end{abstract}

While data on basal metabolic rate (BMR) of adolescents are available (Beirring, 1931; Boothby et al., 1936; Harris and Benedict, 1919), there are only a few studies on the obese (Katch et al., 1985, 1988a,b) and lean (Blunt et al., 1921), and no data that directly compare BMRs of adolescents who differ in body size and composition. The existence of a depressed metabolism would help explain increased rates of body mass gain and continued difficulties in achieving ideal body mass for the obese. We have recently suggested for obese adolescents (Katch et al., $1985,1988 \mathrm{a}, \mathrm{b})$, as others have for obese adults (Segal and Gutin, 1983), an energy conservation hypothesis that results in longterm energy conservation.

In the present paper, we compare the BMR of obese adolescents with normal and lean adolescents within the same age range, but who differ with respect to stature and body composition. The present data lend support to a hypothesis of long-term energy conservation for obese adolescents.

\section{METHODS AND PROCEDURES}

Data for obese adolescents were collected as part of an obesity intervention study (Katch et al., 1985, 1988a,b; Rocchini and Katch, 1986; Becque et al., 1986, 1988; Hattori et al., 1987; Rocchini et al., 1987, 1988). Subjects were recruited via advertisements in local papers and local physician referral. Normal and lean adolescent data were taken from published reports in the literature, and from subjects tested in our laboratory who differed by no more than \pm 2.5 years in age from the obese.

\section{Obese subjects}

Of the 67 obese adolescents only 3 (2 girls and 1 boy) were non-white. All of the obese adolescents had a history of being obese (parental reports). The mean birth mass and body length, determined from hospital records, was $4.67 \mathrm{~kg}(\mathrm{SD}=3.9 \mathrm{~kg})$ and mean

Received July 27, 1989; accepted June 5, 1990 
body length $0.584 \mathrm{~m}(\mathrm{SD}=0.104 \mathrm{~m})$. At the time of testing the mean percent overweight was $179 \%$ of ideal mass for age and stature (Landis, 1988). None of the subjects were under medical care or taking medication. Following written and verbal descriptions of all procedures, parents and children signed informed consent statements.

BMR measurements were made in the Clinical Research Unit of the University Hospital using open circuit spirometry (Katch et al., 1985, 1988b). Following hospital admission, baseline physical examination and an overnight fast, subjects were quietly awakened at 7 A.M. and fitted with a respiratory face mask. A 5 min adjustment period of quiet rest was followed by a $10 \mathrm{~min}$ sampling of expired air that was immediately analyzed for volume with a balanced $0.120 \mathrm{~m}^{3}$ gasometer, percent oxygen with a polargraphic analyzer, and percent carbon dioxide with an infrared detector. The accuracy of measuring minute oxygen uptake $\left(\mathrm{VO}_{2}\right.$ was $\pm 2.0 \%$ (Katch et al., 1985). The $\mathrm{VO}_{2}$ in $\mathrm{m}^{3} \cdot \mathrm{min}^{-1}$ was converted to kilojoules per $\min \left(\mathrm{kJ} \cdot \min ^{-1}\right)$ using the calorific equivalent for oxygen (Carpenter, 1939) determined from the whole body respiratory exchange ratio $\left(\dot{\mathrm{V} C O} \mathrm{CO}_{2} / \mathrm{NO}_{2}\right)$.

Body mass (BM) was measured to the nearest $0.01 \mathrm{~kg}$ with a beam balance scale, stature (HT) to the nearest $0.0001 \mathrm{~m}$ with a stadiometer, and total body volume to the nearest $0.0001 \mathrm{~m}^{3}$ using hydrostatic weighing (Katch et al., 1967). A minimum of 8 to 10 underwater weighing trials were performed and an average of the last 2 to 3 used to represent true underwater weight (Katch, 1969). Residual lung volume (RLV) was determined in duplicate just prior to underwater weighing in the same bent forward seated position, by use of an oxygen dilution procedure (Wilmore, 1969). The average RLV was used in the calculations of total body volume (TBV). Body density $\left(D_{b}\right)$ was converted to percent body fat (\%BF) with the Siri equation (1956), and fat free mass (FFM) was calculated by subtraction, $F F M=B M-$ FM, where FM is fat mass (BM * \%BF). BSA was calculated using the formula of Dubois and Dubois (1916).

\section{Normal subjects}

BMR (closed circuit method), BM, stature, and BSA data for 65 male adolescents (age range $10-15$ yr) reported by Bierring (1931), and 39 female adolescents (age range
10.1-14.3 yr) reported by MacLeod (1924) were used. These studies are unique since complete individual values as well as estimates of measurement error were reported. In all instances reliability exceeded $r=0.92$. Body composition estimates were made using the Mellits and Cheek (1968) and Moore et al. (1963) technique that involves the prediction of total body water (TBW) using gender, age, and stature specific equations. FFM was calculated from TBW, and $\%$ fat determined by subtraction. The validity of these procedures has been previously presented (Sheng and Huggins, 1979; Lukaski and Johnson, 1985).

Additional data on 9 male and 11 female subjects similar in age to the obese, and in body composition to the Beirring and MacLeod subjects were collected in our laboratory and included in the data set. The procedures for BMR and body composition data acquisition were the same as for the obese, with the exception that 6 of the subjects ( 3 male and 3 female) reported to the laboratory at 7 A.M. following an overnight fast. They rested in a bed for 2 hours prior to data collection. With the addition of these subjects the total $\mathrm{N}$ for normal males was 74 , and 50 for normal females.

\section{Lean subjects}

BMR (closed circuit method), BM, stature, and BSA for 14 male and 12 female lean adolescents (age range 7.6-12.0 yr) were taken from the study by Blunt et al. (1921). The classification of underweight was based on mass for age and stature (Landis, 1988), and body composition was estimated as described above. Validity of the BMR exceeded $\mathrm{r}=0.90$ (Blunt et al., 1921). In addition, data on 6 lean males and 6 lean females of the same age as the obese, and with a similar body composition as the Blunt subjects were included in the data set. We used the same data collection methods and procedures as with the obese subjects. Five of the lean subjects ( 3 males and 2 females) did not spend the night in the Clinical Research Unit, but reported to the lab at 7 A.M. and rested for 2 hours prior to data collection. With the addition of these subjects the total $\mathrm{N}$ for the lean group was 20 males and 18 females.

\section{Statistical analyses}

Comparisons between samples were made using a two-way analysis of variance (gen- 
der-by-group). Sheffee's post hoc analysis was used to identify specific mean differences (McNemar, 1969). The relationship between BMR and estimates of body composition were made using linear and polynomial regressions. Comparisons between the slopes were made using analysis of variance. In all cases, data are presented as mean \pm SD. Statistical significance was set at $P \leqslant 0.05$.

\section{RESULTS}

Table 1 presents the data for males and females for each group. There was a significant gender-by-group interaction $(P \leqslant 0.05)$ for all variables except age. Since the lean subjects were slightly younger (ns) than the obese and normal subjects, and since the normal and lean data for the most part were taken from studies conducted many years ago, the possibility of secular effects of growth and nutrition needs to be addressed. There is no way of knowing the extent of this effect on BMR per se; nevertheless, comparisons of the BMRs for the data taken from the literature with the data collected in our laboratory revealed no appreciable or statistical differences in BMR. The greatest difference was $3 \%$ between the BMR data for the 6 lean female adolescents collected in our laboratory, and the female data collected by Blunt et al. (1921). These data suggest that perhaps any secular trends in nutrition and or growth are limited with respect to effects on BMR. Thus, we believe this permits comparisons between groups.

There were significant differences in body composition between the 3 groups for all variables. Moreover, there were gender differences between and within each group for all variables except $B M$ for the obese males and females, and for \% BF and FM for the normal males and females. For all variables, the obese had significantly larger values than the normal and lean, and the normals had larger values than the lean.

Figure 1 presents the BMR data expressed in absolute and relative terms. When expressed as $\mathrm{kJ} \cdot \mathrm{hr}^{-1}$ (Fig. IA), obese males had a nearly $12 \%$ higher BMR compared to normal males $(P \leqslant 0.05)$, and $22 \%$ compared to lean males $(P \leqslant 0.05)$. When indexed to BM (Fig. 1B) obese males had a significantly depressed BMR of $-38 \%$ compared to normal males $(P \leqslant 0.01)$, and $-50 \%$ compared to lean males $(P \leqslant 0.01)$. Similarly, when indexed to FFM (Fig. 1C) and BSA (Fig. 1D), obese males had significantly depressed values of $-16.6 \%(P \leqslant 0.05)$ and $-14 \%(P \leqslant$ $0.05)$ compared to normal males, and $-33 \%$ $(P \leqslant 0.01)$ and $-22.5 \%(P \leqslant 0.01)$ compared to lean males, respectively.

Obese females' BMR expressed as $\mathrm{kJ}$. $\mathrm{hr}^{-1}$ (Fig. 1A) were elevated by $9.5 \%$ $(P \leqslant 0.05)$ compared to normal females, and $23 \%$ compared to lean females $(P \leqslant 0.05)$. As with the males, when indexed to $\mathrm{BM}$ (Fig. 1B) obese females had significantly depressed BMRs of $-34 \%$ compared to normal females $(P \leqslant 0.01)$, and $-53.8 \%$ compared to lean females $(P \leqslant 0.01)$. When indexed to FFM (Fig. 1C), obese female values were depressed by $-11.4 \%$ compared to normal females $(P \leqslant 0.05)$, and $-30 \%$ compared to lean females $(P \leqslant 0.01)$. Indexed to BSA (Fig. 1D), it was less by $-14 \%$ compared to normal females $(P \leqslant 0.05)$, and $-26 \%$ compared to lean females $(P \leqslant 0.05)$.

There were no significant differences in BMR between males and females (Fig. 1). BMR ( $\left.\mathrm{kJ} \cdot \mathrm{hr}^{-1}\right)$ for normal males and females was greater than for lean males and females $(P \leqslant 0.05)$. However, when indexed relative to BM, FFM, and BSA, normals had values that were significantly $(P \leqslant 0.05) \mathrm{de}$ pressed in comparison to lean males and females, respectively.

Table 2 presents the correlations between BMR $\left(\mathrm{kJ} \cdot \mathrm{hr}^{-1}\right)$, age, BM, stature, BSA, and body composition. For females, BSA, BM, and stature accounted for approximately the same amount of variance in $\mathrm{kJ} \cdot \mathrm{hr}^{-1}$ as either FM or FFM. The correlations for males were very similar for obese and lean, while the correlations for normals were much higher for 6 of the 7 comparisons. The correlations with BM, BSA, FFM, and stature were remarkably high for normal males.

\section{DISCUSSION}

The major finding of the present study was the difference in basal metabolic rate between obese, normal, and lean adolescents (Fig. 1). When expressed as $\mathrm{kJ} \cdot \mathrm{hr}^{-1}$ the obese were hypermetabolic. When indexed relative to BM, FFM, or BSA they were hypometabolic. These findings offer conflicting theoretical perspectives in terms of understanding the relationship between obesity, metabolism, and body composition.

The $\mathrm{kJ} \cdot \mathrm{hr}^{-1}$ data suggest that factors other than a depressed metabolism, perhaps hormonal disturbances (Vinik, 1983), increased caloric consumption (Curtis and 


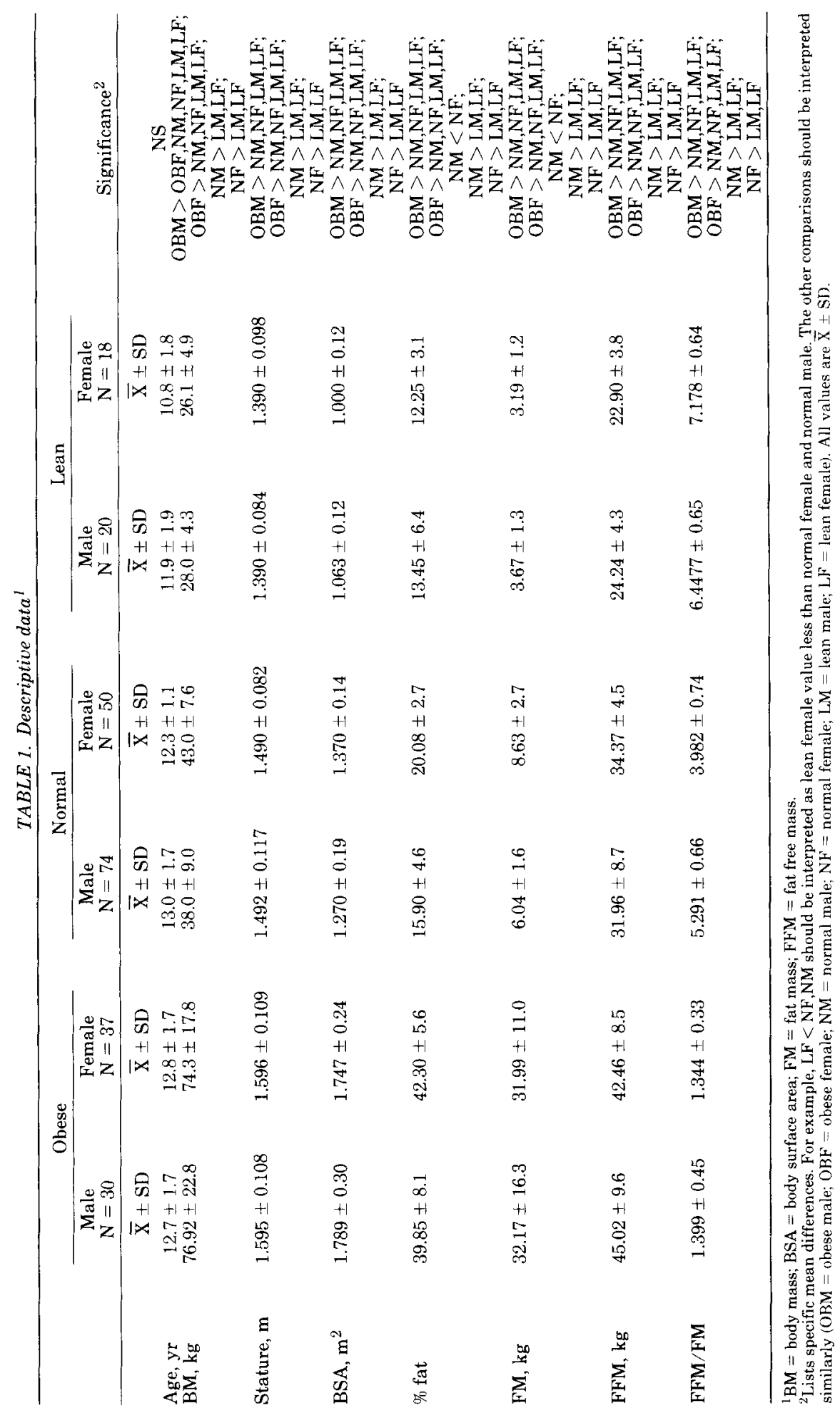



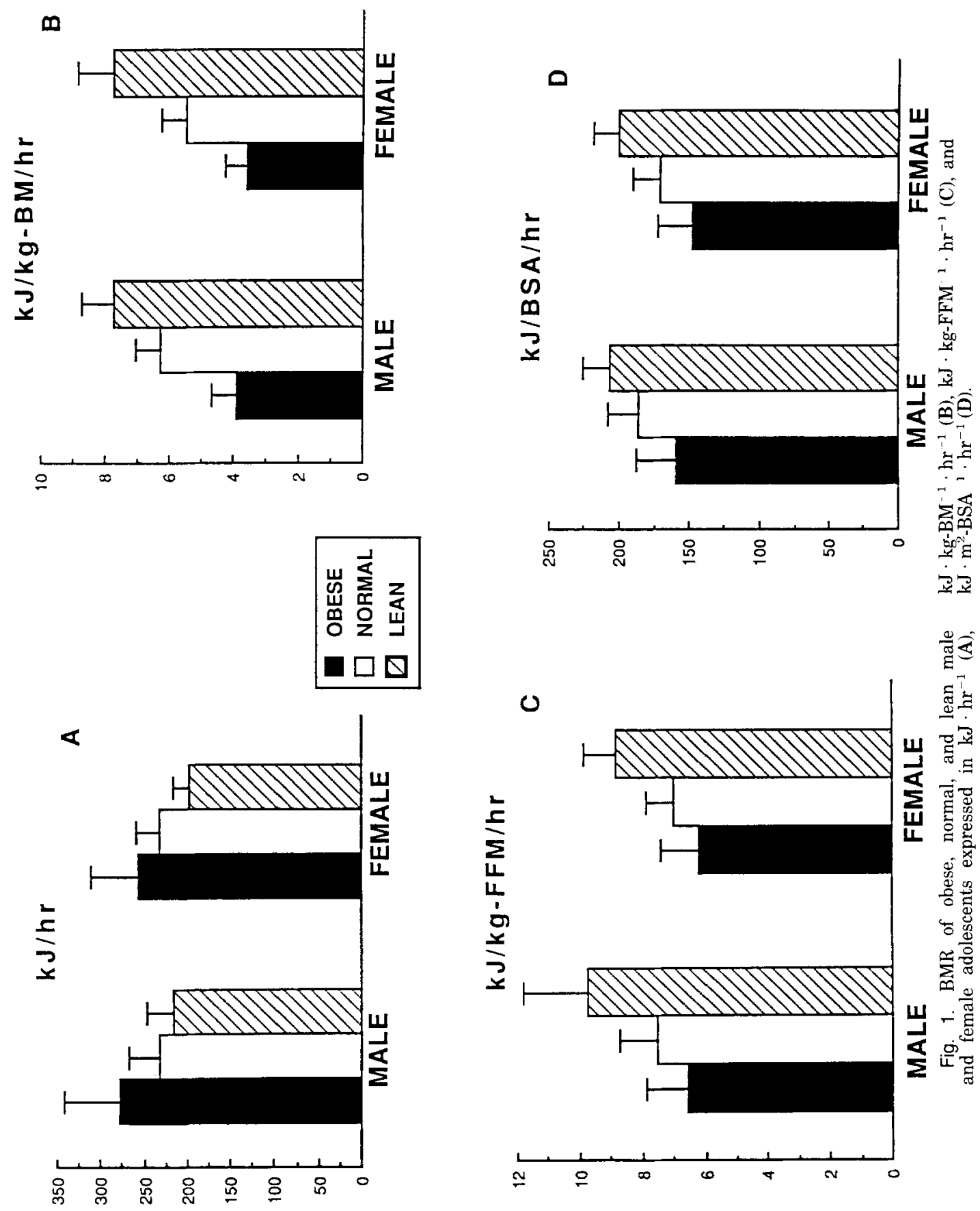
TABLE 2. Correlations between $k J \cdot h r^{-1}$ versus body composition variables

\begin{tabular}{lccccccc}
\hline & \multicolumn{3}{c}{ Male } & & \multicolumn{3}{c}{ Female } \\
\cline { 2 - 5 } kJ $\mathrm{hr}^{-1}$ versus & Obese & Normal & Lean & & Obese & Normal & Lean \\
\hline Age & $0.47^{*}$ & $0.58^{*}$ & $0.52^{*}$ & $0.53^{*}$ & 0.04 & $0.63^{*}$ \\
BM & $0.63^{*}$ & $0.92^{*}$ & $0.52^{*}$ & $0.75^{*}$ & $0.56^{*}$ & $0.74^{*}$ \\
Stature & $0.56^{*}$ & $0.89^{*}$ & $0.58^{*}$ & $0.48^{*}$ & $0.48^{*}$ & $0.77^{*}$ \\
BSA & $0.63^{*}$ & $0.99^{*}$ & $0.58^{*}$ & $0.69^{*}$ & $0.56^{*}$ & $0.76^{*}$ \\
THBF & $0.53^{*}$ & $0.54^{*}$ & 0.37 & $0.43^{*}$ & $0.55^{*}$ & 0.18 \\
FM & $0.59^{*}$ & 0.19 & 0.10 & $0.71^{*}$ & $0.53^{*}$ & 0.50 \\
FFM & $0.41^{*}$ & $0.92^{*}$ & $0.57^{*}$ & $0.64^{*}$ & $0.57^{*}$ & $0.77^{*}$ \\
\hline
\end{tabular}

*Significantly different than zero, $P \leq 0.05$.

Bradfield, 1971), decreased energy expenditure (Bradfield et al., 1971), or a decreased dietary induced thermogenic response to food (Kaplan and Leveille, 1976; Felig and Cunningham, 1983) may be responsible for increased body mass gain in obesity.

In contrast, the ratio expressions of BMR suggest that body mass gain may be more a function of long term energy conservation. The underlying assumption for use of ratio expressions of metabolism have been previously reviewed and shown to be rarely justified on statistical grounds (Tanner, 1949; Katch, 1973). Nevertheless, their use is widespread because they are believed to perform a statistical "adiposectomy," that allows for comparisons of metabolism among individuals or groups that differ in body composition (Cunningham, 1981, 1982; Katch, 1973). The use of ratio expressions is based on the assumption that animals of the same species are comparable, and thus more or less similar. This assumption has been stated as the "Law of Similarity" (Kleiber, 1975), and supposedly offers the basis for comparing build and function of animals of different sizes. The most likely terms for comparing build and function are those that remain constant for changing sizes, such as length $\cdot$ volume ${ }^{-1}$, surface area $\cdot$ volume $^{-1}$ volume $\cdot$ volume ${ }^{-1}$, and volume . mass $^{-1}$. These ratio expressions have long been used in comparative physiology (see Kleiber, 1950, 1975).

There are certain assumptions made when using ratio expressions. In case of the $B M R$, for example, the assumption is that the energy expenditure is directly proportional to $\mathrm{BM}$ (or whatever relative indexing term is used). In fact, the ratio terms assumes the following (Tanner, 1949):

$$
\mathrm{kJ}=¥ \cdot \mathrm{BM}
$$

where the constant $¥$ is determined by the mean body mass and mean energy expenditure in kJ. This ratio, by virtue of its form, implies that it passes through the origin as well as through the mean kJ and BM. Stated another way, the ratio assumes a perfect linear relationship between $\mathrm{kJ}$ and $\mathrm{BM}$, and that the two variables are directly proportional. In actual practice, correlations between kJ and BM (or any other body composition component) are less than $r=1.0$ (see for example, Cunningham, 1982; Tanner, 1949; Katch, 1973). In the present data, no one body composition variable is particularly good as an indexing variable across the 3 groups (Table 2). For example, while BSA is highly correlated to BMR for normal males $(r=0.99)$, it is only moderately correlated $(r \leqslant 0.76)$ for the other groups. The same is true for the correlations with FFM.

As early as 1919 Harris and Benedict were well aware of this ratio "fallacy" and so established the norms of BMR per surface area based on regressed scores (BMR vs. BSA) rather than simple ratio scores (BMR/BSA). In 1938 Benedict stated, "It is believed that far greater progress will be made by discarding all thoughts of a uniformity in heat loss and emphasizing the non-uniformity in heat production" (Benedict, 1938, p. 194). This argues against the use of simple ratio expressions.

Now it is obvious that the daily rate of metabolism (heat production) of a large (obese) individual is greater than that of a smaller (lean) individual. If this metabolic difference is an inherent function of the metabolic requirements of different body tissues (e.g., fat free mass, fat mass), this suggests the need to reference energy metabolism to some measure of composition (Kleiber, 1950). This then is the rationale for use of a 
metabolic-body composition indexing expression. This expression must be a sensitive measure of metabolism per se, and also be able to reflect inherent differences in the energy metabolism of individuals with markedly different fat and fat free components (e.g., males and females, obese and lean, or even the fit and unfit) by considering the specific energy expenditure (thermal equivalents) of both the fat and fat free body mass.

A derived standard with the above characteristics can be written as the sum of the contribution from different tissues (in this example, only fat and fat free mass are considered), each with a specific mass and energy expenditure per unit time, as follows:

$$
\mathrm{kJ} \cdot \mathrm{hr}^{-1}=\left(k_{1} \cdot F M\right)+\left(k_{2} \cdot F F M\right) ;
$$

where $F M$ and $F F M$ are the fat mass and fat free mass in kilograms, and $k_{1}$ and $k_{2}$ are the energy constants for the fat and fat free mass compartments expressed in $\mathrm{kJ} \cdot \mathrm{kg}-\mathrm{FM}^{-1}$. $\mathrm{hr}^{-1}$, and $\mathrm{kJ} \cdot \mathrm{kg}^{-F F M}{ }^{-1} \cdot \mathrm{hr}^{-1}$, respectively. Equation 2 can be rewritten as follows:

$$
\mathrm{kJ} \cdot \mathrm{kg}-\mathrm{BM}^{-1} \cdot \mathrm{hr}^{-1}=\left(\% \mathrm{BF}\left[k_{1}-k_{2}\right]+k_{2}\right)
$$

$$
\mathrm{kJ} \cdot \mathrm{hr}^{-1}=F M \cdot\left(k_{1}+F F M / F M \cdot k_{2}\right)
$$

Equation 3 gives the metabolic rate referenced to body mass, based on the decimal expression of \%BF. Equation 4 considers the metabolic contributions of the fat free mass to fat mass ratio $\left(\mathrm{FFM} \cdot \mathrm{FM}^{-1}\right.$ ). The metabolic rates calculated using these deriva- tions should provide a means of comparing the metabolic rates for individuals of different body masses and compositions taking into consideration the specific energy expenditures of the fat and fat free mass components. For the sake of clarity, these derived values can be referred to as a "theoretical metabolic rate" (TMR) and calculated for each individual (and group) in the present study. To illustrate this derivation, for example, we used a $k_{1}$ constant of $1.117 \mathrm{~kJ} \cdot \mathrm{kg}$ $\mathrm{FM}^{-1} \cdot \mathrm{hr}^{-1}$ and a $k_{2}$ constant of 4.864 $\mathrm{kJ} \cdot \mathrm{kg}-\mathrm{FFM}^{-1} \cdot \mathrm{hr}^{-1}$. These values were recently derived for adults by Garby et al. (1988), and are similar to the values reported by Nakamura and Abe (1975) for adults and more recently by Marks and Katch (1986) for adolescents. It should be understood that these energy constants need to be validated and their use here is only illustrative.

Figure 2 presents the observed BMR and derived TMR values expressed in $\mathrm{kJ} \cdot \mathrm{kg}$ $\mathrm{BM}^{-1} \cdot \mathrm{hr}^{-1}$ (Eq. 3). The large differences between obese, normal, and lean males and females for BMR (solid bars) are obvious (Fig. 1). The differences in TMR (open bars) revealed that the obese male and female values were depressed by an average $22 \%$ compared to the normal and lean males and females $(P \leqslant 0.05)$, respectively. The differences in TMR between the normal and lean males and females was only $8 \%$ (ns). Thus, the TMR, like the BMR expressed relative to BM, BSA, and FFM, was depressed for the obese compared to the normal and lean adolescent. These data suggest an energy conservation hypothesis for the obese adolescent compared to normal and lean adoles-

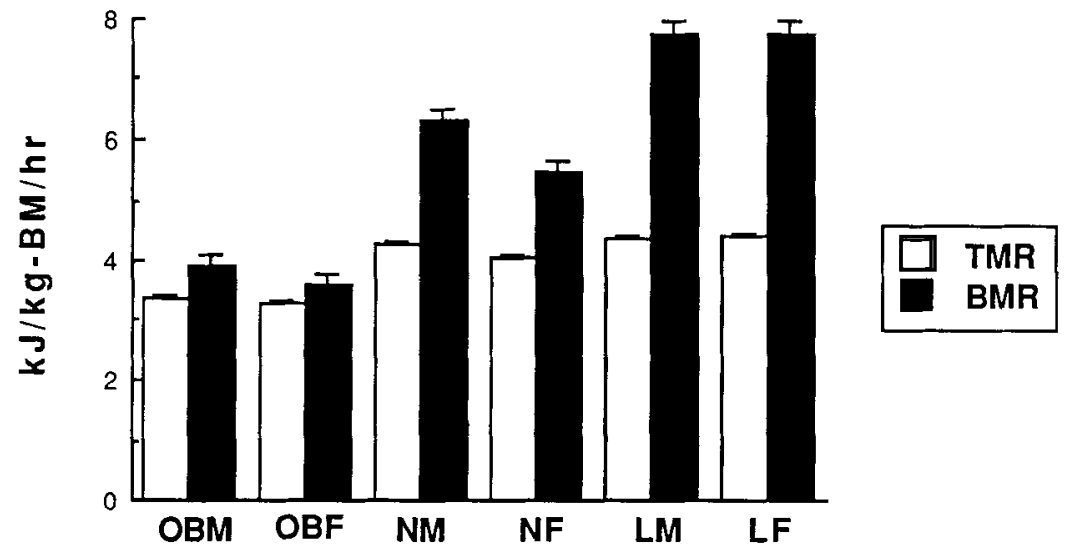

Fig. 2. Comparisons of BMR (filled bars) and TMR (open bars) for obese males (OBM), obese females (OBF),

normal males (NM), and normal females (NF), lean males (LM), and lean females (LF). 
cents. A depressed metabolic rate of $20 \%$ is substantial when converted to energy (fat) storage over time, and could help explain long-term body mass gain for the obese.

In summary, the data from the present study indicate that obese male and female adolescents have depressed resting metabolic rates, and hence conserve energy, compared to normal and lean adolescents whether expressed in traditional ratio terms, or expressed as a theoretical metabolic rate (TMR) that considers the specific thermal equivalents of the fat and fat free body mass. Further research needs to focus on specific mechanisms and factors that contribute to energy conservation for the obese. Specifically, the role that body composition, and in particular the fat free mass, plays in determining individual differences in metabolism appears particularly important, as do changes in the metabolic-FFM relationship.

\section{ACKNOWLEDGMENTS}

Appreciation is expressed to B. Dyer and G. Henry for help in data collection, and to reviewer 0160 for helpful comments and suggestions.

This work was supported by NIH \#Am30989 and CRC \#M01RR0042-21 (University of Michigan).

\section{LITERATURE CITED}

Becque MD, Hattori K, Katch V, and Rocchini AP (1986) Relationship of fat patterning to coronary artery disese risk in obese adolescents. Am. J. Phys. Anthropol. 71:423-429.

Becque MD, Katch V, Rocchini AP, Marks C, and Moorehead C (1988) Coronary risk incidence of obese adolescents: Reduction by exercise plus diet intervention. Pediatrics 81(5):605-612.

Beirring E (1931) The Standard Metabolism of Boys. Copenhagen: Levin and Munksgaard.

Benedict FG (1938) Vital Energetics: A Study in Comparative Basal Metabolism. Washington, DC: Carnegie Inst. Wash Publication.

Blunt K, Nelson A, and Oleson H (1921) Basal metabolism of underweight children. J. Biol. Chem. 49:247-262.

Boothby K, Berkson J, and Dunn H (1936) Studies of the energy of metabolism of normal individuals: A standard for the basal metabolism with a nomogram for clinical application. Am. J. Physiol. 116:468-484.

Bradfield RB, Paulos J, and Grossman L (1971) Energy expenditure and heart rate of obese high school girls. Am. J. Clin. Nutr. 24:1482-1489.

Carpenter TM (1939) Tables, Factors and Formulaes for Computing Respiratory Exchange and Biological Transformation of Energy, Third Edition. Washington, DC: Carnegie Inst Wash.

Cunningham $J$ (1981) Energy expenditure in obesity: Increased metabolic rate in fasting and post-prandial state. Clin. Res. 29:681A.
Cunningham J (1982) Body composition and resting metabolic rate: The myth of feminine metabolism. Am. J. Clin. Nutr. 36:721-726.

Curtis DE, and Bradfield RB (1971) Long-term energy intake and expenditure of obese housewives. Am. J. Clin. Nutr. 24:1410-1417.

Dubois D, and Dubois EA (1916) A formulae to estimate the approximate surface area if height and weight be known. Arch. Intern. Med. 17:863-871.

Felig P, and Cunningham $\mathrm{J}$ (1983) Energy expenditure in obesity in fasting and postprandial state. J. Appl. Physiol. 244:45-51.

Garby L, Garrow JS, Jorgensen B, Lammert O, Madsen K, Sorensen P, and Webster J (1988) Relation between energy expenditure and body composition in man: Specific energy expenditure in vivo of fat and fat free tissue. Eur. J. Clin. Nutr. 42:301-305.

Harris J, and Benedict FA (1919) A Biometric Study of Basal Metabolism in Man. Washington, DC: Carnegie Inst Wash, Publication No. 279.

Hattori K, Becque MD, Katch V, and Rocchini AP (1987) Fat patterning of adolescents. Ann. Hum. Biol. 14(1):23-28

Kaplan MD, and Leveille GA (1976) Calorigenic response in obese and non-obese women. Am. J. Clin. Nutr. 29:1108-1113.

Katch FI (1969) Practice curves and errors of measurement in estimating underwater weight by hydrostatic weighing. Med. Sci. Sports Exerc. 1:212-216.

Katch FI, Michael ED, and Horvath SM (1967) Estimation of body volume by underwater weighing: Description of a simple method. J. Appl. Physiol. 23:811-813.

Katch V (1973) Use of the oxygen/body weight ratio in correlational analyses: Spurious correlations and statistical considerations. Med. Sci. Sports Exerc 5(4):253-257.

Katch V, Rocchini A, Marks C, Becque MD, and Moorehead C (1985) Basal metabolism of obese adolescents: Age, gender and body composition effects. Int. J. Obes. 9:69-76.

Katch V, Becque MD, Marks C, Moorehead C, and Rocchini AP (1988a) Oxygen uptake and energy output during walking of obese male and female adolescents. Am. J. Clin. Nutr. 47(1):26-32

Katch V, Becque MD, Marks C, Moorehead C, and Rocchini AP (1988b) Basal metabolism of obese adolescents: Inconsistent diet and exercise effects. Am. J. Clin. Nutr. 48(3):565-569.

Kleiber M (1950) Physiological meaning of regression equations. J. Appl. Physiol. 2:417-423.

Kleiber M (1975) The Fire of Life, 2nd Edition. Huntington, NY: Krieger Publishing.

Landis RJ (1988) National Health and Nutrition Examination Survey II (NHANES II) data tape. School of Public Health, University of Michigan.

Lukaski HC, and Johnson PE (1985) A simple, inexpensive method of determining total body water using a tracer dose of $\mathrm{D}_{2} \mathrm{O}$ and infrared absorption of biological fluids. Am. J. Clin. Nutr. 41:363-370

MacLeod G (1924) Studies of the normal basal energy requirement. Doctoral Dissertation, Columbia University.

Marks C, and Katch V (1986) The standard metabolic rate: A model of body composition and energy balance. Midwest Medical Convention. Ann Arbor, Mi (Abstract).

McNemar Q (1969) Psychological Statistics. Fourth Edition. New York: John Wiley and Sons, Inc.

Mellits ED, and DB Cheek (1968) Growth and body water, chapter 9. In DB Cheek (ed.): Human Growth: 
Body Composition, Cell Growth, Energy and Intelligence. Philadelphia, Pa.: Lea and Febiger, pp. 135-149.

Moore FD, Olesen KH, McMurrey JK, Parker HV, Ball MR, Boyden CM (eds.) (1963) The body cell mass and its supporting environment: Body composition in health and disease. Philadelphia: WB Saunders.

Nakamura M, and Abe K (1975) Energy constants for adipose and fat free tissue. Xth International Congress of Nutrition, Kyoto, Japan (Abstract \#1102).

Rocchini AP, and Katch V (1986) Role for aldosterone in blood pressure regulation of obese adolescents. Am. J. Cardiol. 57:613-618.

Rocchini AP, Katch V, Shork A, and Kelch R (1987) Insulin and blood pressure during weight loss in obese adolescents. Hypertension 10:167-173.

Rocchini AP, Katch V, Anderson J, Henderliter J, Becque MD, Martin M, and Marks C (1988) Blood pressure in obese adolescents: The effects of weight loss. Pediatrics 5:45-62.
Segal KR, and Gutin B (1983) Thermic effects of food and exercise in lean and obese women. Metabolism 32(6):581-589

Sheng HP, and Huggins RA (1979) A review of body composition studies with emphasis on total body water and fat. Am. J. Clin. Nutr. 32:630-647.

Siri WE (1956) Gross composition of the body. In JH Lawrence and CA Tobias (eds.): Advance in Biological and Medical Physics IV. New York: Academic Press.

Tanner JM (1949) Fallacy of per-weight and per-surface area standards and their relation to spurious correlations. J. Appl. Physiol. 2:1-15.

Vinik AI (1983) Gastrointestinal hormones and nutrient balance. In: BC Hansen (ed.): Controversies in Obesity. Vol. 5. Metabolism. New York: Praeger Publ., pp. $15-40$.

Wilmore JH (1969) A simplified method for determination of residual lung volumes. J. Appl. Physiol. $27: 96-100$. 